\title{
AVALIAÇÃO PRELIMINAR DO QUESTIONÁRIO DE INFORMÁTICA EDUCACIONAL (QIE) EM FORMATO ELETRÔNICO
}

\author{
Maria Cristina Rodrigues Azevedo Joly \\ Márcia Adriana Silveira
}

\begin{abstract}
RESUMO. O objetivo deste estudo foi analisar a eficácia do Questionário de Informática Educacional (QIE) quanto à compreensão das questões, à objetividade do enunciado e às características da tarefa solicitada aos professores. Participaram 21 docentes do ensino superior particular, dos quais 57,1\% eram do sexo feminino e 76,2\% possuíam formação pedagógica. O questionário é eletrônico e tem quatro módulos, visando à caracterização do sujeito, conceito, aplicação e frequiência do uso de recursos tecnológicos aplicados ao processo de ensino-aprendizagem. Os resultados indicaram a necessidade de reformulação do módulo II, constituído por questões abertas, em função da dificuldade de compreensão e realização da tarefa pelos sujeitos. Os módulos III e IV, compostos por questões fechadas, não apresentaram dificuldades e permaneceram iguais. O novo formato do QIE será testado em pesquisas futuras, com amostra mais representativa
\end{abstract}

Palavras-chave: avaliação informatizada; instrumento eletrônico; informática educacional.

\section{EDUCATIONAL INFORMATICS QUESTIONNAIRE: PRELIMINARY EVALUATION}

\begin{abstract}
The Informatics Educational Questionnaire was analyzed to prove its efficacy in respect to comprehension questions, proposition objectivity and task features, besides identifying the possibility of technological resources variety of uses in the teaching - learning process. The questionnaire was answered by 21 professors from private universities, among them $57.1 \%$ were female and $76.2 \%$ had pedagogical background. The questionnaire was electronic and comprised by four modules aimed to the subjects' characterization, the concepts, application and use of the technological resources. The data analysis pointed to the need of module II reformulation due to the comprehension and the tasks performing difficulties by the subjects. The other modules did not reveal any difficulties to the subjects.
\end{abstract}

Key words: electronic evaluation; electronic instrument; educational informatics.

\section{INTRODUÇÃO}

O conceito de tecnologia educacional, segundo Pfromm Netto (2001), refere-se tanto a um instrumental a serviço do ensino e aprendizagem designado como recurso tecnológico quanto às mudanças que se processam no aprendiz, envolvendo teoria, pesquisa e desenvolvimento de recursos físicos, mentais e comportamentais. Os recursos tecnológicos, como, por exemplo, a televisão, o videocassete, o videodisco, o DVD, o computador, são materiais e equipamentos mecânicos, eletromecânicos, eletrônicos e informatizados. Sua função primeira é a de apoio pedagógico à aprendizagem, gestão e desenvolvimento educacional, em função de suas possibilidades de interatividade.

Nos Estados Unidos estão sendo desenvolvidos projetos e estudos voltados à reorganização de currículos, capacitação do corpo docente e reestruturação dos laboratórios de Informática Educacional. Esses projetos apresentam como objetivos comuns tornar o professor hábil no uso da tecnologia, para assim melhorar o processo de aprendizagem do aluno e o seu desempenho acadêmico. Isto posto, possibilitará ao aluno ser criativo para solucionar problemas e tomar decisões,

\footnotetext{
* Doutora em Psicologia pelo Instituto de Psicologia da Universidade de São Paulo. Docente da Universidade de São Franscisco.

Endereço para correspondência: Rua Alexandre Rodrigues Barbosa, 45, CEP 13251040, Itatiba-São Paulo.

Email: cristinajoly@ saofrancisco.edu.br

\# Mestre em Psicologia pela Universidade São Fransico. Docente da Universidade São Fransico.
} 
bem como selecionar informações de uma variedade de fontes, processar e relatar resultados e comunicarse usando os recursos informatizados (Fowler, 2001; Distad, 2001; Vitale, 2001; Baldwin, 2001; Bitter, 2001; Bedford, 2001).

As pesquisas americanas, nessa área, iniciaram-se com uma caracterização do uso dos recursos tecnológicos aplicados à educação por parte dos professores para, posteriormente, analisar, dentre os padrões de desempenho necessários para atuar com a informática educacional, quais recursos o professor efetivamente usa em sua prática pedagógica. (National Center for Education Statistics, 2000; Starr, 2000; ISTE,1999).

A Sociedade Internacional para Tecnologia em Educação (International Society for Technology in Education - ISTE) elaborou, a partir de uma pesquisa de levantamento realizada com uma amostra representativa de docentes de todos os níveis de ensino, uma lista dos padrões de desempenho necessários ao professor para o trabalho com informática educacional. Foram elencados padrões relativos à operação, uso e avaliação de recursos tecnológicos (multimídia, hipermídia e telecomunicações), instalação e aplicação de programas (softwares) para o uso didático e ferramentas de produtividade (editor de textos, planilhas, base de dados, etc), avaliação, seleção e integração da informática educacional ao currículo. Além disso, os resultados apontaram que cabe também ao professor ser capaz de atuar e discutir sobre o uso da informática educacional com eqüidade e ética, respeitando os critérios impostos pela legislação (ISTE, 1999; Marinho, 2002).

No tocante à realidade brasileira, a maioria dos relatos disponíveis sobre o uso de recursos tecnológicos refere-se a experiências educacionais aplicadas (Sampaio \& Leite, 1999; Grings \& Mallmann, 2000; Gomes \& Vermelho, 2001). Na perspectiva de Moran (2001), é necessária a preparação de professores não somente para incorporar a informática como mais um recurso educacional disponível, mas também para pesquisar, com a finalidade de coletar a maior quantidade possível de informações relativas a conceitos, opiniões, crenças e aplicações educacionais, dentre outras, que caracterizem como os professores conceituam e usam os recursos tecnológicos disponíveis na educação.

Geralmente, estas pesquisas de levantamento ou surveys são realizadas através de um questionário, que, segundo Günther (1999), é tido como o principal instrumento no levantamento de dados por amostragem. Questionário é definido como um conjunto de perguntas sobre um determinado tema que visa medir opinião, interesses ou aspectos de personalidade e colher informações biográficas do sujeito.

O objetivo da pesquisa é determinante para a seleção de conceitos e itens a serem utilizados na construção do questionário, relacionando-os diretamente à população-alvo pretendida e à amostra selecionada. O contexto social da aplicação do instrumento (situação e local, experiência e referências do pesquisador e conhecimentos do sujeito) e sua estrutura lógica quanto à seleção e organização dos elementos (questões e itens) devem ser também considerados (Coolican, 1999; Günther, 1999).

Faz-se necessário destacar, segundo Fowler (1998), Günther (1999) e Coolican (1999), que um bom item é aquele que possibilita ao sujeito compreendê-lo em suas várias dimensões (conceito, linguagem, expectativas de resposta) para respondê-lo com disposição e veracidade, evitando-se aqueles que possibilitem interpretações com viés ou ambigüidade. Dessa forma, cada item deve ser específico, breve, claro, objetivo, e ser redigido com vocabulário correto, preciso, apropriado à pesquisa (termos técnicos) e à amostra. A escolha do tipo de pergunta é essencial tanto para a estrutura lógica do instrumento quanto para o custo de resposta exigido dos sujeitos. As perguntas abertas são essenciais numa pesquisa exploratória, quando se quer verificar a abrangência das respostas, apesar de implicarem alto custo para o respondente, por exigirem maior esforço, principalmente em questionários auto-aplicados, o que pode diminuir a probabilidade de o sujeito completar a tarefa. As perguntas fechadas, por sua vez, são indicadas para investigar temas mais pesquisados e também conhecidos pelos sujeitos, principalmente quando os respondentes são muitos e dispõe-se de pouco tempo.

Acrescente-se aos aspectos anteriormente descritos que a padronização das questões e itens é feita em função do tipo de aplicação do questionário, podendo este, segundo Günther (1999), ser aplicado através de entrevista pessoal ou telefônica e autoaplicado (via postagem, correio eletrônico ou internet). Dentre esses tipos de aplicação, algumas considerações serão feitas para o questionário autoaplicado via internet, por ser o instrumento em análise no presente estudo.

São incontestáveis algumas vantagens quando se usa a informática com instrumentos de qualidade, podendo-se destacar: rapidez na coleta de informações, ampliação da amostra com facilidade de 
acesso, grande possibilidade de armazenamento de diferentes tipos de informações em banco de dados com processamento e análise ágil e precisa, economia, segurança e interatividade com o sujeito. A despeito dos aspectos positivos, há alguns negativos, que podem ocorrer e devem, portanto, ser considerados. Caberia destacar: impossibilidade de acesso a computadores e/ou internet por uma grande parte da população; falta de familiaridade com esses equipamentos e recursos; falta de segurança do instrumento e das informações; qualidade técnica insuficiente, mesmo quando o instrumento é validado e padronizado; problemas de interpretação e compreensão, sem possibilidade de interação imediata com o aplicador, implicando, na maioria das vezes, em interrupção da tarefa (Kumar, 1996; Anastasi \& Urbina, 2000).

Além disso, cumpre considerar que o uso de instrumentos informatizados ainda é pouco comum, podendo suscitar resistências, ansiedade e desconfiança por parte dos respondentes (Adánez, 1999). O fato implica diminuição, em torno de 50\%, dos instrumentos respondidos por completo, o que, conseqüentemente, reduz a amostra. Apesar disso, Günther (1999, p.255) afirma que "baixas taxas de respostas significam não necessariamente baixo grau de representatividade, especialmente no caso de amostras probabilísticas".

O termo "teste informatizado" só deve ser utilizado, segundo Adánez (1999), quando sua organização e aplicação são feitas por computador ou via internet. Devese priorizar, para a construção do instrumento, além dos aspectos válidos para questionários impressos, já descritos, o uso de computadores rápidos e com recursos apropriados ao objetivo da investigação, conectados a provedores seguros. Deve-se também definir uma estrutura lógica simples e objetiva, no que se refere à apresentação de instruções e itens, à forma de responder, ao tempo de apresentação dos itens e à formatação (tela, letra, cores).

Enfatize-se, à guisa de síntese, a necessidade de sempre se realizar um estudo piloto para verificar se a estrutura e organização do questionário, principalmente em formato eletrônico, atende aos objetivos da pesquisa que se pretende realizar, pois esse instrumento será usado como fonte prioritária de coleta de dados.

Como citado, novas tecnologias de informação e comunicação se inseriram no processo tradicional de ensino-aprendizagem. Diante desse fato, se faz necessário avaliar como o professor usa os recursos tecnológicos em sua prática e a possibilidade de desenvolver um instrumento eletrônico para testar a eficiência de um método mais operacional e rápido. Inserido nesse contexto, o presente estudo tem como objetivo analisar a eficácia do Questionário de Informática Educacional (QIE) em formato eletrônico quanto à estrutura, no que se refere à compreensão das questões, objetividade do enunciado e características da tarefa solicitada ao respondente.

\section{MÉTODO}

\section{Sujeitos}

Participaram da pesquisa 21 professores que trabalhavam em instituições particulares de ensino superior da região de Campinas/SP. Os sujeitos possuíam especialização (2), mestrado(11) e doutorado(8), sendo $57,1 \%$ do gênero feminino e $76,2 \%$ deles com formação pedagógica.

\section{Material}

Foi utilizado na presente pesquisa o Questionário de Informática Educacional (QIE), em formato eletrônico e auto-aplicado via Internet. Para tanto, o questionário foi dividido em quatro módulos:

- Módulo I - Dados pessoais: identificação do sujeito, formação acadêmica, experiência docente, tipo de instituição em que leciona e em qual área de conhecimento atua - Exatas, Humanas ou Biológicas;

- Módulo II - Opinião sobre recursos tecnológicos: são cinco questões abertas,colocadas abaixo, em que o professor deve colocar palavras que definam quais são seus conceitos e opinião acerca do uso da tecnologia de informação no processo de ensinoaprendizagem:

1. Cite três recursos tecnológicos que poderiam ser utilizados em sala de aula.

2. Cite três alternativas do uso da tecnologia para facilitar o processo de aprendizagem.

3. Cite três alternativas do uso da tecnologia para avaliar seus alunos.

4. Cite três meios que lhe possibilitariam adquirir as habilidades necessárias para utilizar as ferramentas tecnológicas.

5. Cite três habilidades que você acredita serem adquiridas pelos alunos quando o professor usa a tecnologia.

- Módulo III - Tipo de uso dos recursos tecnológicos: composto por questões fechadas (sim ou não), que questiona o uso de recursos tecnológicos em suas aulas, além de investigar como o professor os aplica na prática pedagógica e qual suporte institucional ele recebe para isso, como descrito a seguir: 
1- Você usa recursos tecnológicos em suas aulas?

SIM ( ) NÃO( )

Se sua resposta foi SIM:

Cite as três ferramentas tecnológicas mais utilizadas por você em suas aulas:

Você mesmo utiliza as ferramentas tecnológicas para preparar e desenvolver suas aulas?

SIM ( ) NÃO ( ) Quem o faz por você?

Se sua resposta foi NÃO:

Você gostaria de utilizar ferramentas tecnológicas?

SIM ( ) NÃO ( )

2- Caso você queira usar recursos tecnológicos em sua aula:

2.1- Você tem os equipamentos disponíveis na Instituição?

SIM ( ) NÃO( )

Se sua resposta foi SIM:

2.2- Você tem acesso livre a esses equipamentos na Instituição? SIM ( ) NÃO ( )

2.3- Você tem suporte institucional para usar esses equipamentos?

$\operatorname{SIM}($ ) NÃO( )

- Módulo IV - Freqüência de uso dos recursos tecnológicos: este módulo verifica a frequiência de uso dos recursos tecnológicos e mídia pelo professor. As possibilidades de resposta foram organizadas em uma Escala do tipo Likert (0 a 3 pontos):

1. Marque para cada item abaixo sua freqüência de uso da tecnologia em geral

\begin{tabular}{|l|l|l|l|l|}
\hline Situações de uso & Nunca & Algumas vezes & Muitas vezes & Sempre \\
\hline Uso pessoal (Ex: em casa, bancos, etc) & & & & \\
\hline Cursos de capacitação & & & & \\
\hline Atividades em sala de aula & & & & \\
\hline Atividades administrativas na escola & & & & \\
\hline Atividades de pesquisa e/ou ensino & & & & \\
\hline
\end{tabular}

O QIE foi elaborado considerando-se estudos realizados por Dirksen \& Tharp (2000), Zhao \& Coll. (2001) e Dewert (2000) relacionados aos padrões de desempenho em informática educacional definidos pelo ISTE (2000).

\section{3- Procedimento}

Uma versão preliminar do QIE foi elaborada em papel e testada com quatro professores universitários, para verificar sua viabilidade como instrumento de avaliação dos conteúdos pretendidos. Os resultados revelaram haver compreensão da tarefa requerida por parte dos sujeitos, definindo-se, a partir das sugestões dadas quanto ao vocabulário e à estrutura de questões, a versão eletrônica do instrumento disponibilizada na
Internet para ser respondida pelos sujeitos. Após a resposta a cada módulo, os sujeitos eram solicitados a avaliá-lo considerando as três variáveis: compreensão da questão, objetividade do enunciado e características da tarefa. A avaliação do Módulo II foi feita em relação a cada questão. Exemplo: Avalie essa questão quanto a: Compreensão da Questão: sim ( ) não ( ); Instruções do Enunciado: objetivo ( ) confuso ( ) Tarefa solicitada: fácil ( ) difícil ( )

Procedeu-se à coleta dos dados por 30 dias consecutivos, sendo armazenados em um banco de dados do tipo MySOL tanto o consentimento dos participantes para ser sujeito de pesquisa como os dados obtidos.

\section{RESULTADOS}

Procedeu-se à análise estatística da avaliação feita pelos sujeitos em cada módulo, considerando as variáveis: compreensão da questão, objetividade do enunciado e características da tarefa. Definiu-se: compreensão da questão como a forma de o sujeito entender o enunciado para respondê-la; características da tarefa como sua classificação, por parte do sujeito, como fácil ou difícil, ao responder ao questionário; objetividade do enunciado como sua classificação, pelo sujeito, como objetivo ou confuso.

As questões analisadas foram 1, 2, 3, 4 e 5 do módulo II e questão 1 do módulo III.

Tabela 1. Análise das questões do Módulo II segundo os aspectos avaliados pelos sujeitos

\begin{tabular}{|c|c|c|c|c|c|}
\hline Questão & Aspectos & Alternativas & $\%$ de respostas & Qde Cochran & $p$ valor \\
\hline \multirow[t]{6}{*}{1} & Compreensão & Não & $4,8 \%$ & 0,50 & 0,7788 \\
\hline & & Sim & $95,2 \%$ & & \\
\hline & Enunciado & Confuso & $4,8 \%$ & & \\
\hline & & Objetivo & $95,2 \%$ & & \\
\hline & Tarefa & Difícil & $9,5 \%$ & & \\
\hline & & Fácil & $90,5 \%$ & & \\
\hline \multirow[t]{6}{*}{2} & Compreensão & Não & 19,0 & 4,75 & 0,0930 \\
\hline & & Sim & 81,0 & & \\
\hline & Enunciado & Confuso & 42,9 & & \\
\hline & & Objetivo & 57,1 & & \\
\hline & Tarefa & Difícil & 33,3 & & \\
\hline & & Fácil & 66,7 & & \\
\hline \multirow[t]{6}{*}{3} & Compreensão & Não & 23,8 & 5,09 & 0,0784 \\
\hline & & Sim & 76,2 & & \\
\hline & Enunciado & Confuso & 33,3 & & \\
\hline & & Objetivo & 66,7 & & \\
\hline & Tarefa & Difícil & 52,4 & & \\
\hline & & Fácil & 47,6 & & \\
\hline \multirow[t]{6}{*}{4} & Compreensão & Não & 23,8 & 4,00 & 0,1353 \\
\hline & & Sim & 76,2 & & \\
\hline & Enunciado & Confuso & 42,9 & & \\
\hline & & Objetivo & 57,1 & & \\
\hline & Tarefa & Difícil & 42,9 & & \\
\hline & & Fácil & 57,1 & & \\
\hline \multirow[t]{6}{*}{5} & Compreensão & Não & 19,0 & 6,89 & 0,0319 \\
\hline & & Sim & 81,0 & & \\
\hline & Enunciado & Confuso & 23,8 & & \\
\hline & & Objetivo & 76,2 & & \\
\hline & Tarefa & Difícil & 47,6 & & \\
\hline & & Fácil & 52,4 & & \\
\hline
\end{tabular}


Pode-se observar, na Tabela 1, que os sujeitos consideraram compreensíveis as questões $1,2,3$, e 4 do módulo II, o enunciado objetivo e a tarefa fácil. $\mathrm{Na}$ questão 5, a avaliação da tarefa teve julgamentos muito próximos, possibilitando assim uma diferença estatisticamente significante entre as três variáveis avaliadas. Esse resultado indicou a necessidade de revisar essa questão, de modo a atender ao seu objetivo de investigação.

$\mathrm{Na}$ análise do percentual de sujeitos por aspecto avaliado em cada questão, verificou-se que, apesar de não terem sido encontradas diferenças significantes entre eles, ficaram evidentes dificuldades quanto ao enunciado e tipo de tarefa solicitado, exceção feita à questão 1 , na qual parece não terem sido evidenciados problemas em relação a nenhuma das variáveis. Na questão 4 há uma porcentagem alta de respostas negativas em relação ao enunciado e tarefa, revelando, assim, a necessidade de se modificar o texto da questão, a fim de facilitar sua compreensão e, conseqüentemente, a tarefa de respondê-la.

Participaram também da análise do instrumento dois juízes independentes, os quais identificaram se as respostas dos sujeitos eram as esperadas ou não, em relação aos possíveis usos e aplicações dos recursos tecnológicos na educação, como estratégias metodológicas, conforme descritos na literatura ( Quadro I).

Quadro 1. Critério de resposta para análise dos módulos pelos juízes independentes.

\begin{tabular}{|l|l|}
\hline $\begin{array}{l}\text { Módulo / } \\
\text { Questão }\end{array}$ & \multicolumn{1}{|c|}{ Critério } \\
\hline II - 1 & $\begin{array}{l}\text { Recurso Tecnológico: Equipamentos Multimídia } \\
\text { (Computador, Gravador, Retroprojetor, TV), Internet } \\
\text { (Correio Eletrônico, Rede, Listas de Discussão) }\end{array}$ \\
\hline II -2 & $\begin{array}{l}\text { Aplicação Recurso na Aprendizagem: pesquisa na Internet, } \\
\text { EAD, produção /análise de filmes, simulação, uso de } \\
\text { correio eletrônico, etc }\end{array}$ \\
\hline II - 3 & $\begin{array}{l}\text { Aplicação Recurso na Avaliação: prova eletrônica, uso de } \\
\text { internet para pesquisa, criação de páginas, elaboração de } \\
\text { banco de dados com resultados de desempenho, etc }\end{array}$ \\
\hline II -4 & $\begin{array}{l}\text { Capacitação Docente: cursos, leitura, orientação de } \\
\text { parceiros, etc }\end{array}$ \\
\hline II -5 & $\begin{array}{l}\text { Habilidade do Aluno: autonomia, seleção de informação, } \\
\text { solução de problemas, etc }\end{array}$ \\
\hline III -1 & $\begin{array}{l}\text { Recurso Tecnológico: Equipamentos Multimídia } \\
\text { (Computador, Gravador, Retroprojetor, TV), Internet } \\
\text { (Correio Eletrônico, Rede, Listas de Discussão) }\end{array}$ \\
\hline
\end{tabular}

$\mathrm{O}$ critério de análise utilizado pelos juízes independentes referiu-se ao tipo da resposta dada pelo sujeito em relação à avaliação feita por ele da questão. Cada resposta foi pontuada: como coerente - C $(1,0)$ quando atendia ao critério estabelecido no
Quadro I, sua avaliação em compreensão indicava facilidade e em enunciado assinalava objetividade; e como incoerente - I $(0,0)$ quando não atendia ao critério estabelecido no Quadro I e, a despeito disso, sua avaliação em compreensão indicava facilidade e em enunciado assinalava objetividade. Por exemplo: a resposta dada à questão II - 2 era uso de software de simulação e, se a avaliação indicasse compreensão da questão e objetividade do enunciado, a resposta do sujeito seria pontuada como coerente pelo juiz; caso a resposta dada à questão II - 2 fosse computador, por exemplo, e se a avaliação indicasse compreensão da questão e objetividade do enunciado, a análise do juiz seria resposta incoerente do sujeito.

A ANOVA de Friedman e o Coeficiente de Kendall de concordância foram aplicados na análise feita pelos dois juízes independentes em relação à coerência de respostas dadas pelos sujeitos em sua avaliação dos itens de cada módulo, segundo os critérios estabelecidos (Quadro I).

Tabela 2. Comparação dos resultados da análise dos juízes independentes com a análise dos sujeitos em relação à compreensão das questões do módulo II

\begin{tabular}{|c|c|c|c|c|c|}
\hline \multirow[b]{2}{*}{ Questão } & \multirow[b]{2}{*}{ Comparação } & \multicolumn{2}{|c|}{$\%$ de respostas } & \multirow[b]{2}{*}{$Q$ de Cochran } & \multirow[b]{2}{*}{$p$ valor } \\
\hline & & Sim & Não & & \\
\hline \multirow[t]{2}{*}{1} & Sujeitos & $95,2 \%$ & $4,8 \%$ & 0,34 & 0,5637 \\
\hline & Juiz & $90,5 \%$ & $9,5 \%$ & & \\
\hline \multirow[t]{2}{*}{2} & Sujeitos & $81,0 \%$ & $19,0 \%$ & 6,40 & 0,0114 \\
\hline & Juiz & $42,9 \%$ & $57,1 \%$ & & \\
\hline \multirow[t]{2}{*}{3} & Sujeitos & $76,2 \%$ & $23,8 \%$ & 4,45 & 0,0348 \\
\hline & Juiz & $42,9 \%$ & $57,1 \%$ & & \\
\hline \multirow[t]{2}{*}{4} & Sujeitos & $76,2 \%$ & $23,8 \%$ & 0,34 & 0,5637 \\
\hline & Juiz & $81,0 \%$ & $19,0 \%$ & & \\
\hline \multirow[t]{2}{*}{5} & Sujeitos & $81,0 \%$ & $19,0 \%$ & 0,14 & 0,7055 \\
\hline & Juiz & $76,2 \%$ & $23,8 \%$ & & \\
\hline
\end{tabular}

A análise das respostas dadas pelos sujeitos no Módulo II ( Tabela 2) em relação àquelas esperadas pelos dois juízes independentes, considerando-se os critérios explicitados no Quadro1, revelou que apenas aquelas relativas às questões 1 e 4 do módulo II correspondiam às esperadas. As demais questões $(2,3$ e 5) apresentaram várias respostas imprevisíveis. Verificou-se, de acordo com o coeficiente de correlação de Pearson ( $r=0,68 ; p=0,0007)$, a existência de concordância entre os juízes na avaliação das respostas e nas avaliações feitas pelos sujeitos, sendo possível inferir, com um nível de confiança de $95 \%$, que os sujeitos não compreenderam as questões 2 e 3, uma vez que a comparação realizada entre a avaliação dos juízes e a dos sujeitos é estatisticamente significante $(\mathrm{p}<0,05)$. 
À guisa de síntese, levando-se em conta a avaliação dos sujeitos e a dos juízes, observou-se a necessidade de reformulação do Módulo II, pois esse não atendeu aos objetivos pretendidos pelo instrumento no formato de questões abertas como foi inicialmente organizado.

Tabela 3. Avaliação das questões do Módulo III, segundo os aspectos avaliados pelos sujeitos

\begin{tabular}{|c|c|c|c|c|c|}
\hline \multirow{2}{*}{$\frac{\text { Questão }}{1}$} & Aspectos & \multicolumn{4}{|c|}{ Alternativas \% de respostas $Q$ de Cochranp valor } \\
\hline & \multirow{2}{*}{ Compreensão } & Não & $0,0 \%$ & 4,00 & 0,1354 \\
\hline & & Sim & $100,0 \%$ & & \\
\hline & \multirow[t]{2}{*}{ Enunciado } & Confuso & $9,52 \%$ & & \\
\hline & & Objetivo & $90,48 \%$ & & \\
\hline & \multirow[t]{2}{*}{ Tarefa } & Difícil & $9,52 \%$ & & \\
\hline & & Fácil & $90,48 \%$ & & \\
\hline \multirow[t]{6}{*}{2} & \multicolumn{2}{|c|}{ Compreensão Não } & $0,0 \%$ & 4,67 & 0,0970 \\
\hline & & Sim & $100,0 \%$ & & \\
\hline & \multirow[t]{2}{*}{ Enunciado } & Confuso & $9,52 \%$ & & \\
\hline & & Objetivo & $90,48 \%$ & & \\
\hline & \multirow[t]{2}{*}{ Tarefa } & Difícil & $14,29 \%$ & & \\
\hline & & Fácil & $85,71 \%$ & & \\
\hline
\end{tabular}

As questões do Módulo III, diferentemente das do Módulo II, foram bem compreendidas, assim como o enunciado e a tarefa solicitada considerados fáceis pela maioria dos sujeitos (Tabela 3).

Tabela 4. Avaliação pelos sujeitos das questões do Módulo IV, segundo o aspecto tarefa

\begin{tabular}{|c|c|c|c|c|}
\hline Questão & Tarefa & $\%$ de respostas & $Q$ de Cochran & $p$ valor \\
\hline \multirow[t]{2}{*}{1} & Difícil & $20,0 \%$ & & \\
\hline & Fácil & $80,0 \%$ & 2,00 & 0,5724 \\
\hline \multirow[t]{2}{*}{2} & Difícil & $13,3 \%$ & & \\
\hline & Fácil & $86,7 \%$ & & \\
\hline \multirow[t]{2}{*}{3} & Difícil & $13,3 \%$ & & \\
\hline & Fácil & $86,7 \%$ & & \\
\hline \multirow[t]{2}{*}{4} & Difícil & $20,0 \%$ & & \\
\hline & Fácil & $80,0 \%$ & & \\
\hline
\end{tabular}

Quanto às respostas dadas no Módulo IV (Tabela 4), identificou-se muita facilidade por parte dos sujeitos em realizar a tarefa, havendo compreensão. Não se verificaram diferenças estatisticamente significantes entre as variáveis avaliadas em todo o módulo ( $\mathrm{p}=0,5724)$.

Identificou-se, de modo geral, que apenas o Módulo II, dos quatro organizados no instrumento, requer modificações. Esses resultados obtidos serão discutidos à luz da literatura, relevando-se o fato de o instrumento ser auto-aplicado e eletrônico, respondido independentemente da possibilidade de qualquer orientação de um aplicador.

\section{DISCUSSÃO}

A análise estatística do questionário indicou a necessidade de reformulação das questões abertas que avaliavam conhecimento específico, pois estas se mostraram de mais difícil compreesão, principalmente as que compunham o Módulo II. Apesar de justificarse tecnicamente a utilização das questões abertas para uma pesquisa exploratória (Günther,1999), como se tem por objetivo utilizá-las , faz-se necessária a restruturação do QIE para que possa ser considerado um instrumento eletrônico de avaliação eficaz.

Os resultados revelaram que muitos sujeitos, neste módulo, tiveram dificuldade não somente com a tarefa, mas também com a avaliação das questões. Em contrapartida, as questões fechadas (Módulo III) e as que se utilizaram de escala Likert (Módulo IV) foram respondidas adequadamente pelos sujeitos, e sua avaliação revelou clareza, objetividade e compreensão. Tanto as questões com escolha entre duas alternativas quanto a escala do tipo Likert, com opção de quatro níveis de resposta para cada item, facilitam, segundo Coolican (1999), a tarefa do respondente, por apresentarem um grande número de afirmações balanceadas em relação às atitudes a serem analisadas, possibilitando diferentes níveis de respostas.

Cabe destacar também que a dificuldade do sujeitos em compreender os conceitos presentes nas questões por falta de conhecimento técnico é um aspecto relevante a ser considerado, em relação à estrutura do questionário e à população a que se destina. Apesar de esse dado não ser diretamente explicitado pelos sujeitos, pode-se inferi-lo ao avaliarem-se as respostas dadas a cada um dos itens. Esse resultado constata a necessidade de avaliação de um questionário, quando usado como instrumento de avaliação, no tocante às relações que se estabelecem entre a correspondência dos conceitos a serem avaliados com itens/tipos de questões selecionados e a capacidade de compreensão e interpretação desses itens e conceitos pela população pesquisada ( Coolican, 1999; Pasquali, 1999, Anastasi \& Urbina, 2000).

É importante assinalar que, atualmente, com o avanço da tecnologia da informação e da globalização, a realização de pesquisas de levantamento é muito facilitada quando se faz uso de instrumentos eletrônicos. Várias são as vantagens desse uso, podendo-se destacar: rapidez na transmissão, armazenamento e análise de dados, facilidade de acesso a pessoas de diferentes regiões geográficas e apresentação atrativa e interativa do instrumento ao 
respondente, visando facilitar seu maior envolvimento com a tarefa e concluí-la (Cronbach, 1996; Pasquali, 1999; Anastasi \& Urbina, 2000). Não se pode contar, apesar disso, com a total confiabilidade nas conexões feitas às redes de computadores, pois essas são, na maioria das vezes, muito lentas e inconstantes, correndo-se o risco de perder dados ou obtê-los de forma incompleta, como ocorreu, na presente pesquisa, com 19 sujeitos que não responderam a todos os módulos.

A questão da segurança de acesso às informações é algo pouco discutido ou não discutido cientificamente, mas vale lembrar que instrumentos eletrônicos, quando disponibilizados via Internet, devem estar alojados em portais seguros e confiáveis, a fim de que sejam respeitadas as normas técnicas e éticas de qualquer tipo de avaliação.

Além disso, observou-se que, em se tratando de um questionário auto-aplicado via Internet, sem possibilidade de interação imediata com o aplicador, responder a questões conceituais abertas pode ter sido uma tarefa difícil para os sujeitos. Considerando-se essa hipótese como verdadeira, esta atividade implicou em alto custo para os sujeitos, tendo sido, provavelmente, por este motivo que alguns deles (19) não completaram o preenchimento do questionário. Tal fato foi também observado em estudos realizados por Kumar (1996).

As reformulações propostas neste estudo serão realizadas no Questionário de Informática Educacional, para que ele seja posteriormente validado como amostra representativa da população docente, vindo a ser utilizado como instrumento de coleta de dados que possibilita a definição de um perfil do professor quanto ao uso da tecnologia no ensino brasileiro. Isto posto, será possível identificar os recursos tecnológicos que estão sendo incorporados à prática pedagógica e avaliar suas implicações no processo de aprendizagem, viabilizando-se uma inserção adequada da informática educacional nos currículos acadêmicos.

\section{REFERÊNCIAS}

Adánez, G.P. (1999) Procedimientos de construccion y analisis de testes psicometricos. Em S. M. Wechsler e R. S. L. Guzzo (Orgs.), Avaliação psicológica: perspectiva internacional.(pp.57-100). São Paulo: Casa do Psicólogo.

Anastasi, A. \& Urbina, S. (2000). Testagem psicológica. Porto Alegre: Artmed.

Baldwin, A. (2001). Collaboration, inquiry, and reflection united with instructional technology. Disponível em:

$<$ http://www.pt3.org/pt3_grants/project_db/project_desc.php3? grahntu_id=368>. (Acessado em 15/11/2001)

Bedford, J. (2001). Preparing tomorrow's teachers to use technology: the institute for the advancement of student centered learning. Disponível em: <http://www.pt3.org/ pt3_grants/project_db/project_desc.php3?grantu_id=386>. (Acessado em 10/2/2002)

Bitter, G. G. (2001). The e-learning anytime anywhere, NETS digital video library for preparing tomorrow's teachers to connect curriculum with tecnology. Disponível em: <http://www.pt3.org/pt3_grants/project_db/project_desc.ph p3 ?grantu_id=358>. (Acessado em 15/1/2002)

Coolican, H. (1999). Research methods and statistics in psychology. London: Hodder / Stoughton Educational.

Cronbach, L. J. (1996) Fundamentos da testagem psicológica. Porto Alegre: Artes Médicas.

Dewert, M. H. (2000) Preparing tomorrow's teachers to use technology: asking the right questions about essential conditions. Journal of Computing in Teacher Education 16(3), 3-5.

Dirksen, D. J. \& Tharp, M. D. (2000) Moving beyond the Crossroads: teachers as agents for change. [Trabalho completo] Em International Society of Educacional Technology (Org.). NECC 2000 Research Proceedings "Connecting @ The Crossroads" (p. 26 -36 ) Eugene: ISTE.

Distad, L. (2001). Teachers as leaders in the use of technology Disponível em: <http://www.pt3.org/ pt3_grants/project_db/project_desc.php3 ?grantu_id=4 32>. (Acessado em 5/3/2002)

Fowler, F. L. (1998) Design and evaluation of survey questions. Em L. Bickman e D. J. Rog (Eds.), Handbook of applied social research methods (pp. 343-374). Thousand Oaks, CA:Sage.

Fowler, W. (2001). Technology integration in education initiative (TIE-IN). Disponível em: <http:// www.pt3.org/pt3_grants/project_db/project_desc.php3?gran tu_id=395>. (Acessado em 15/1/2001)

Gomes, P.V.\& Vermelho, S. C. (2001, agosto). A experiência da PUCPR na implantação das tecnologias de informação e comunicação no Ensino Superior. Colabor@- Revista Digital da CVA - RICESU, 1 (1). Disponível em: <http://http://gemini.ricesu.com.br/colabora/n1/index1.htm>. (Acessado em 28/04/2003)

Grings, E. S. \& Mallmann, M. (2000). Ambiente virtual de aprendizagem: uma experiência interdisciplinar no Ensino Superior. Disponível em: <http://www.pt3.org/ pt3_grants/projects_db/project_desc.php3?grantu_id=398>. (acessado em 15/11/2001)

Günther, H. ( 1999) Como elaborar um questionário. Em Pasquali, L. (Org.) Instrumentos psicológicos: manual prático de elaboração (pp.231-258) Brasília: LabPAM/IBAPP.

International Society For Technology In Education (1999) National Educational Technology Standards for Teachers. Eugene: ISTE.

Kumar, D. (1996) Computer and assessment in science education. Disponível em: http://www.ed.gov/databases/ ERIC_digest/ed395770.html ..(Acessado em 20/09/2002).

Marinho, S. P. (2002). Tecnologia, educação contemporânea e desafios ao professor. Em M. C. R. A Joly (Org.), 
Tecnologia no ensino: implicações para aprendizagem (pp.41-64). São Paulo: Casa do Psicólogo.

Moran, J. M. (2001). Ensino e aprendizagem inovadoras com tecnologias audiovisuais e telemáticas. Em J. M. Moran., M. T. Masetto e M. A. Behrens (Orgs.), Novas tecnologias $e$ mediação pedagógica. (pp. 11-66) São Paulo: Papirus.

National Center for Education Statistics. (2000) Report on Teacher and Computers. Disponível em: <http://www.education-world.com/a_tech/tech026.shtml >. (Acessado em 15/11/2001)

Pfromm Netto, S. (2001). Telas que ensinam: média e aprendizagem: do cinema ao computador. Campinas: Alínea.

Pasquali, L.(1999). Instrumentos psicológicos: manual prático de elaboração. Brasília: LabPAM/IBAPP.

Sampaio, M.N., e Leite, L.S. (1999). Alfabetização tecnológica do professor. Rio de Janeiro: Vozes.

Starr, L. (2000). Fact, Fiction, or Opinion? Evaluating Online Information. Disponível em: $<$ http://www.educationworld.com/a_curr/curr194.shtml>. (Acessado em 15/11/2001)

Vitale, M. (2001). Infusing assistive technology into teacher preparation programs. Disponível em: <http://www.pt3.org/pt3_grants/ptoject_db/project_desc.ph p3?grantu_id=430>. (Acessado em 5/1/2002)

Wall, J. E. (2000) Technology- delivered assessment: diamonds or rocks? ERIC Clearinghouse on Couseling and Students Services. Disponível em: <http://www.ed.gov/databases/ERIC_Digests/ed446325.htm 1>. (Acessado em 20/09/2002)

Zhao, Y., Byers, J., Mishra, P., Topper, A., Chen, H., Enfield, M., Ferdig, R., Frank, K., Pugh, K. e Hueysantan, S. (2001) What do they know? A comprehensive portrait of exemplary technology: using teachers. Journal of Computing in Teacher Education 17(2) p. 24-36.

Recebido em 10/12/2003

Revisado em 25/03/2003

Aceito em 30/05/2003 